\title{
Selecting from dynamic environments: Attention distinguishes between blinking and moving
}

\author{
Yaïr Pinto, Christian N. L. Olivers, and Jan Theeuwes \\ Vrije Universiteit Amsterdam, Amsterdam, The Netherlands
}

\begin{abstract}
Pinto, Olivers, and Theeuwes (2006) showed that a static target can be efficiently found among different types of dynamically changing distractors. They hypothesized that attention employs a broad division between static and dynamic information, a hypothesis that conforms with earlier research. In the present study, we investigated whether attention can only make use of this crude division or can exploit more subtle discriminations within the dynamic domain. In Experiment 1, participants were able to efficiently find a blinking target among moving distractors and moving targets among blinking distractors, although all items changed at the same rate and produced the same change in local luminance. In Experiment 2, search for a dynamic target among dynamic distractors was aided when we gave the distractors additional dynamic cues. Experiment 3 showed that making the displays equiluminant affected search efficiency for a static target among moving distractors, but not among blinking distractors. The findings refute the broad division hypothesis and suggest that object continuity plays an important role in selection.
\end{abstract}

From everyday experience it is clear that dynamic stimuli stand out. Examples of this are the flashing lights on an ambulance and your friend's waving when you are looking for him in a crowd. Both are examples of stimuli intended to attract attention. Research has confirmed the intuitive notion that dynamic stimuli can guide or capture attention (McLeod, Driver, \& Crisp, 1988; Watson \& Humphreys, 1995; Yantis \& Jonides, 1984).

The reverse case is harder to appreciate intuitively. What is the fate of a static item in a constantly changing environment? Would the dynamic elements in the environment continuously compete for your attention, making it very difficult to find the static object? Or would dynamic surroundings allow for a relatively efficient segmentation of the static object from its background? And, important in the present study, would this be different for different types of dynamics, such as blinking and motion?

\section{Efficient Search in Dynamic Scenes}

To investigate whether static objects can indeed guide attention, Theeuwes (2004) asked participants to determine the orientation of a static bar among distractor bars that were all abruptly changing. In one condition, all distractor bars changed in a single frame from horizontal or vertical to either left or right oblique (by $45^{\circ}$; therefore, after the change, the target was the only horizontal or vertical bar). In another condition, in addition to changing orientation, the distractors also disappeared from their old locations and abruptly reappeared at new, random locations. The key finding was that search was much more efficient (as indicated by small or even absent set size effects) than in a control condition in which all items (including the distractors) were static. Theeuwes argued that attention does not prefer a dynamic item per se, but the item that differs the most from its surroundings; in a dynamic environment, this is the unique static item.

However, Davis and Leow (2005) have argued that it is actually not the general distinction between dynamic and static that allows for efficient search, but that, more specifically, motion is the crucial factor. They reasoned that Theeuwes's (2004) displays allowed apparent motion to emerge as the items changed from one orientation or position to the other. Davis and Leow suggested that a static item among items with other dynamic features, such as luminance changes or onsets, may not be found efficiently. In support of this, they found that search was highly inefficient for a static target among distractors that did not move, but did among those that abruptly change in both color and luminance (without disappearing). They concluded that filtering on the basis of motion may have a special status, whereas filtering a single static item from a set of items carrying dynamic properties other than motion (such as abrupt luminance changes) may be very difficult.

Pinto, Olivers, and Theeuwes (2006) investigated this issue further by conducting a series of experiments in which participants searched for a vertical or horizontal line segment among tilted line segments. In the control condition, all line segments were static. In the crucial 
conditions, the target was static, but the distractors behaved dynamically; they either all moved or all continuously produced abrupt onsets and offsets (were blinking). Relative to the control condition, in which all distractors were static, search was efficient regardless of whether the distractors were blinking or moving. In fact, performance with blinking and moving distractors turned out to be remarkably similar. Control experiments showed that the efficient search of the static target among blinking distractors was not due to either average or momentary luminance differences, nor to long-range apparent motion. In other words, and contrary to Davis and Leow's (2005) findings, the results provided evidence that a static item is efficiently found in a dynamic environment, without these dynamics necessarily involving motion.

The question then remained why Pinto et al. (2006) found efficient search with blinking distractors, whereas Davis and Leow (2005) failed to do so. To resolve this issue, Pinto et al. adapted Davis and Leow's procedure by presenting dynamic distractors that were abruptly changing in luminance without disappearing. The result was that search for the static target became inefficient. Apparently, attention can only effectively distinguish between static and blinking stimuli when the blinking objects completely disappear, suggesting that the static/dynamic segmentation is at least partly object-based, rather than purely luminance based.

\section{A Broad Static/Dynamic Division?}

On the basis of their results, Pinto et al. (2006) argued that attention makes use of a broad division of the visual scene into static information on the one hand, and dynamic information on the other. The dynamic channel is broadly defined, involving motion and blinking, but possibly also other changes related to these two, such as looming. Search for a static target among dynamic distractors or vice versa then becomes efficient, because attention can turn to one type of representation and largely ignore the other. Note here that Pinto et al. did not find completely parallel search for the static target (completely flat search slopes). This might be due to a default attentional set, which makes it easier for participants to attend to dynamic rather than stationary items. This view is supported by the findings of Müller and von Mühlenen (1999), who found search for a static target among moving distractors to become very efficient only after intensive training, whereas the reverse yielded efficient search without training. Support for such a broad division of visual information into a dynamic and static channel also comes from physiological data. It has been shown that there are two subsystems in the brainmagnocellular and parvocellular. The processing of dynamic aspects of stimuli takes place mainly in the magnocellular system, whereas static properties are preferentially analyzed in the parvocellular system (see, e.g., Breitmeyer \& Ganz, 1976; Livingstone \& Hubel, 1988; Zeki et al., 1991).

An important question then is whether the attentional system can make efficient use only of this broad division between static and dynamic stimuli or whether it can differentiate between the two different types of dynamic stimuli. In other words, are all dynamic stimuli treated alike by at- tention, or can their representations be separated and guide attention in different ways? Even though Davis and Leow (2005) may not have been right in stating that static stimuli can be segmented only from motion stimuli, and not from luminance onsets, they may still be right in that the attention system differentiates between motion and onsets.

At the very lowest level in the processing stream, cells should treat blinking and moving objects equally because both types of stimuli involve the same luminance changes within the cells' receptive fields (at least in the luminancebased apparent motion displays used by Pinto et al., 2006). Also higher up, there appear to be cells in V1 that respond in the same manner to both moving and blinking items (Andersen, 1997). Furthermore, it has been shown that adaptation to blinking stimuli leads to impaired motion perception, and vice versa (Chapman, Hoag, \& Giaschi, 2004; Green, 1981). This too suggests that, at least at the lower perceptual levels, the two types of dynamic stimuli are processed within a common pathway (presumably the magno pathway).

But also at an attentional level, it has been proposed that there is a general dynamic dimension driving attention (Folk, Remington, \& Wright, 1994). Folk et al. presented participants with an irrelevant precue followed by a target display. They found interference of the precue with target processing only when precue and target were either both statically or both dynamically defined. That is, when the target was a moving item or an onset, both moving and onset precues interfered. However, when the target was a color singleton, a moving precue did not interfere, and vice versa. As Folk et al. put it: "With respect to the architecture of attentional control, the present results provide support for the hypothesis that attentional control settings may be established for only very broad stimulus categories associated with the distinction between static and dynamic discontinuities" (p. 327). Thus, Folk et al.'s results support the notion that, preattentively, the visual scene is separated into dynamic and static stimuli and participants can prioritize either of these categories. Furthermore, their results suggest that the attention system is not able to differentiate between the two different types of dynamic transients.

\section{Separate Motion and Onset Systems}

There are also studies that cast doubt on the hypothesis that motion and onsets are treated alike. On a trivial note, the visual system must eventually treat motion and onset stimuli differently, because, after all, they look different. The important question is at what level the representations start to diverge, and whether attention can make use of this divergence.

Physiologically the picture is not entirely clear. It seems that, until V1, both motion and onsets may be treated alike. After that, processing diverges, with moving stimuli being processed in areas such as V5/MT (Albright, 1984; Zeki, 1974). The fate of onsets is less clear. There are some studies that suggest that motion derivatives such as flicker are also processed in MT (O'Keefe \& Movshon, 1998), but there is also evidence for onset sensitivity in the lateral intraparietal sulcus (Gottlieb, Kusunoki, \& Goldberg, 1998). Zihl, von Cramon, and Mai (1983) reported a patient with bilateral lesions in motion-sensitive areas, who 
had difficulty distinguishing movement, but who had little trouble with detecting flicker.

Also, on a functional level, there is evidence that suggests that attention is able to distinguish between motion and onsets. Yantis and Jonides (1984) asked participants to search for a target letter among distractor letters. When the target letter appeared with an abrupt onset, search slopes were independent of set size, indicating that the abrupt onset captured attention. In a similar design, Hillstrom and Yantis (1994) presented target letters that were in motion to participants. Several versions of moving stimuli were tested, including oscillation, looming, and nearby moving contours. None of these moving stimuli captured attention. This suggests that motion and onsets are treated differently by the attention system. However, Hillstrom and Yantis's findings are not undisputed. Franconeri and Simons (2003) argued that moving items do capture attention, but that this can be masked by simultaneous offsets. Franconeri and Simons found that several forms of motion captured attention when masking was controlled for. The debate has not ended, as findings by von Mühlenen, Rempel, and Enns (2005) suggest that, whereas onsets appear to capture attention regardless of the simultaneous presence of other dynamics, other types of changes require a static environment in order to capture attention.

\section{The Present Study}

The results so far indicate that when a static item is searched, dynamic transients are relatively easily ignored by the visual system. There seems to be a fundamental distinction between the processing of static stimuli and dynamic transients in the brain, which suggests that these results can be generalized. However, some studies suggest that, within the dynamic channel, attention can select distinct subcategories, such as onsets.

If, in the case of search for a static object in a dynamic background, attention can indeed only operate on a broad division between static and dynamic items, this yields several general predictions. The first prediction is that it should be hard to find a dynamic object in a dynamic environment, even if the exact type of dynamics differ. Second, when participants are searching for a static item, dynamic distractors should be equally easy to ignore regardless of whether they are moving or blinking. The first prediction was tested in the first two experiments, in which we looked at search for blinking targets among moving distractors, for moving targets among blinking distractors, and for moving targets among distractors that were both blinking and moving. Both experiments suggested a distinction between motion and onsets. Furthermore, some research suggests that luminance plays a crucial role in motion detection, but not in onset detection. For example, Cavanagh, Tyler, and Favreau (1984) found perceived velocity to be a weighted average of luminance and chrominance velocity information. Gegenfurtner and Hawken (1995) showed that determining motion direction for drifting sinusoidal gratings was impaired at low temporal frequencies when luminance cues were removed. As a comparison, Yantis and Hillstrom's (1994) findings suggest that the onset of a new object captures attention regardless of whether this onset is accompanied by a change in luminance. The third experiment therefore sought to further dissociate the motion and onset transient systems by investigating search efficiency among moving and blinking distractors under equiluminant conditions.

\section{EXPERIMENT 1 Blinking Item Among Moving Distractors}

If attention employs only a broad division between static and dynamic stimuli, then it should be difficult to find a dynamic target among dynamic distractors, just as it is difficult to find a static target among static distractors. This should be the case regardless of whether the dynamics are of the same or of a different type - other things, such as frequency and local saliency of change, being equal. If, however, motion and flicker are differently encoded at an early enough level for attention to distinguish between different types of dynamic stimuli, it should be easier to find a target that differs in the type of dynamics from the background.

To test these predictions, we set up an experiment with four conditions (see Figure 1 for example displays). Participants searched for a moving or blinking target among either moving or blinking distractors. Motion was defined by an intersected vertical line jumping back and forth between two positions. Blinking was established by having an identical stimulus turn on and off. The rate and abruptness of the change was equal for the blinking and moving conditions. The only difference between the two types of dynamics was that in the case of motion, the line abruptly disappeared and reappeared somewhere else for a certain period, whereas in the blinking condition it disappeared and then reappeared after the same period. Therefore, the local luminance change at an item's location was identical in both cases. Because, by definition, blinking items switch off for a brief period, one could argue that observers at any moment in time only need to search half the number of items in the display, in comparison with the motion displays. To control for this, the blinking conditions included set sizes that doubled those of the motion conditions. Thus, given the controls for the frequency of change, the saliency of the local luminance change, and the overall set size, the only difference between the conditions was the quality of the dynamics (i.e., blinking vs. motion). If attention can distinguish blinking from moving, then search should be more efficient in the blinkingamong-moving condition or the moving-among-blinking condition than in the conditions where both target and distractors were moving or blinking.

\section{Method}

Participants. Twelve participants ranging in age from 17 to 35 years, average 23.0 years, took part as paid volunteers. All participants completed all of the conditions. All had normal or correctedto-normal vision.

Apparatus and Stimuli. The experiment was conducted on a computer with a Pentium IV processor, a 17-in. monitor, and a standard QWERTY keyboard. The software package E-Prime was used for the presentation and timing of the experimental trials. The stimulus field consisted of a $9 \times 8$ imaginary matrix $\left(17.26^{\circ} \times 12.59^{\circ}\right.$ visual angle). In its cells, white (CIE $x, y$ coordinates, 0.283, 0.302; 
A

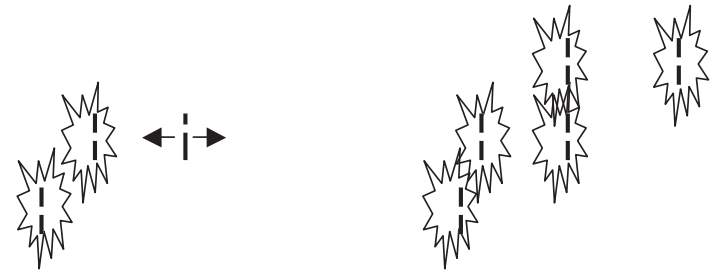

3n
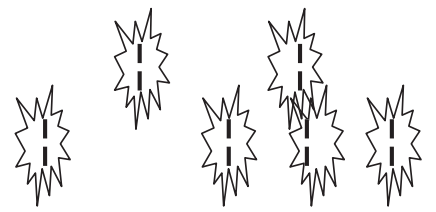

$\left\{\begin{array}{l}M \\ 1 \\ 4 k\end{array}\right.$

B
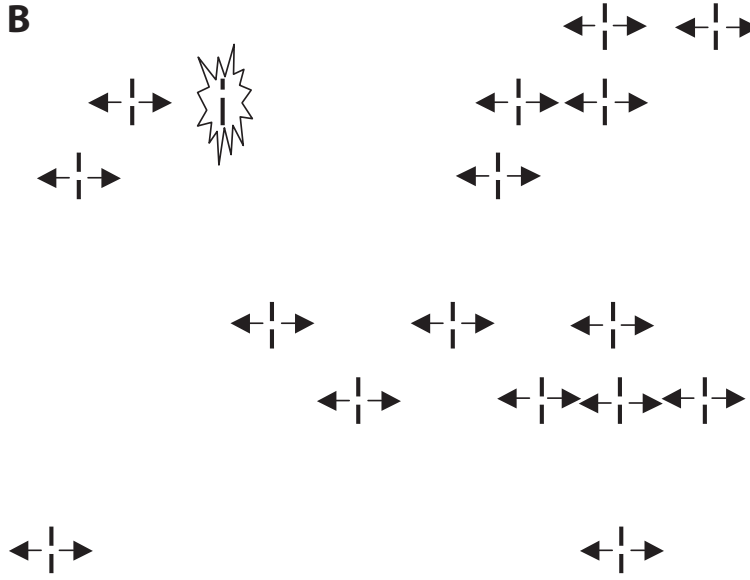

Figure 1. Typical examples of the search displays used in Experiments 1 and 3 . Participants searched for a line segment with a gap above or below the middle among line segments with a gap in the middle. In Experiment 1, the line segments were white and the background was black. Experiment 3 also featured green lines on an equiluminant gray background. The "flash" surrounding an item indicates that it was blinking; the arrows indicate that it was moving. Panel A depicts an example of a trial in which the target was moving and the distractors were blinking (set size 16). Panel B depicts an example of a trial in which the target was blinking and the distractors were moving (set size 16).

luminance, $71.86 \mathrm{~cd} / \mathrm{m}^{2}$; background, $0 \mathrm{~cd} / \mathrm{m}^{2}$, as measured with a Tektronix photometer) line segments of size $0.87^{\circ}$ with a gap in the middle (size $0.12^{\circ}$ ) and one white line segment with a gap above or below the middle (distance from the middle $0.17^{\circ}$ ) were placed in the center of the cells with a horizontal jitter of $\pm 0.58^{\circ}$. The distractors could appear anywhere on the $9 \times 8$ matrix; the target could appear anywhere except in the middle (row 5, column 4 or 5).

Design and Procedure. Participants sat approximately $90 \mathrm{~cm}$ from the monitor, with their fingers resting on the " $\mathrm{k}$ " and " $\mathrm{m}$ " keys, which were used as the response buttons. The experiment consisted of 20 blocks, each containing 36 trials. The order of the blocks was repeated every 4 blocks and followed a Latin square design. Each sequence of 4 blocks corresponded to 4 main conditions. In all conditions, participants searched for a target with a gap either above or below the middle of the line among distractors (with a gap in the middle of the line). In the blinking-among-moving condition, participants searched for a blinking target that moved back and forth among distractors, between its original location and a location $0.32^{\circ}$ to the right of this location. This gave a strong impression of apparent motion. The target changed after a random period between 150 and $300 \mathrm{msec}$, with a $25 \%$ chance of switching on or off after $150 \mathrm{msec}, 25 \%$ after $200 \mathrm{msec}, 25 \%$ after $250 \mathrm{msec}$, and $25 \%$ after $300 \mathrm{msec}$. The same held true for the distractor elements, which moved after a period between 150 and $300 \mathrm{msec}$, with a $25 \%$ chance of flipping after $150 \mathrm{msec}, 25 \%$ after $200 \mathrm{msec}, 25 \%$ after $250 \mathrm{msec}$, and $25 \%$ after $300 \mathrm{msec}$. All blinking items started "on" (visible). Furthermore, the chances of turning on and off were uncorrelated between the items and were uncorrelated to the previous onset/offset time. The same held true for the moving items, since the time before an item changed position was uncorrelated between items and uncorrelated to previous times it took before it moved. In the blinking-among-blinking condition, participants looked for a blinking target among blinking distractors (blinking in the same way as in the blinking-among-moving condition). In the movingamong-blinking condition, participants looked for a moving target that moved in the same way as the moving distractors in the previous conditions among distractors that blinked on and off-in the same way as the blinking distractors in the previous two conditions. In the moving-among-moving condition, participants looked for a moving target among moving distractors (moving in the same way as in the other conditions). In the conditions with moving distractors, set sizes varied randomly within a block between 9,17 , and 33 (i.e., 8,16 , or 32 distractors plus one target); in the conditions with blinking distractors, set sizes varied randomly within a block between $9,17,33$, and 65 (i.e., 8, 16, 32, or 64 distractors, plus one target) to control for the fact that blinking items disappear for half the time (on average). The task was to determine the location of the gap within the target element. Participants pressed " $k$ " and " $m$ " for gaps above and below the middle of the line, respectively. Before each block, text appeared on the screen, telling participants which condition followed. Participants were instructed that both speed and accuracy were important. The first 4 blocks were disregarded as practice. The other 16 blocks were included in the analyses. The experiment took approximately $60 \mathrm{~min}$, with breaks between the blocks.

\section{Results and Discussion}

Overall, error percentages were low (see Table 1). A two-way ANOVA with condition (blinking-among-moving, blinking-among-blinking, moving-among-moving, and moving-among-blinking) and set size $(9,17$, and 33) revealed a main effect of condition $\left[F(3,33)=8.62, M S_{\mathrm{e}}=\right.$ $0.003, p<.001]$, a main effect of set size $[F(2,22)=$ $\left.11.69, M S_{\mathrm{e}}=0.005, p<.001\right]$, and a significant interaction $\left[F(6,66)=10.41, M S_{\mathrm{e}}=0.002, p<.001\right]$. More errors were made when all items were of the same type of dynamics than when target and distractors were defined by different types of dynamics. Errors also increased with set size, but again more so when all items were of the same type of dynamics. The error pattern resembled the response time (RT) pattern, and there were no speed-accuracy trade-offs. We will therefore concentrate on the mean RTs of the correct trials.

Trials on which RTs were 2.5 standard deviations away from the mean of the respective cell were excluded from analysis, resulting in a loss of approximately 5\% of the trials. Figure 2 shows RTs as a function of set size for each condition, as well as the accompanying search slope. A two-way ANOVA on mean RT for each participant with condition (blinking-among-moving, blinking- 
Table 1

Average Error Percentages for the Different Conditions and the Different Set Sizes of Experiments 1-3

\begin{tabular}{lccr}
\multicolumn{3}{c}{ Different Set Sizes of Experiments 1-3 } \\
\cline { 2 - 4 } & 9 & 17 & 33 \\
\cline { 2 - 4 } & & & \\
& 2.39 & 2.39 & 4.82 \\
Experiment 1 & 1.25 & 2.33 & 7.66 \\
Moving-blinking & 3.71 & 1.80 & 3.42 \\
Moving-moving & 2.13 & 6.21 & 18.44 \\
Blinking-moving & & & \\
Blinking-blinking & 2.73 & 5.07 & 6.72 \\
Experiment 2 & 3.50 & 2.70 & 3.54 \\
Control & 2.40 & 2.38 & 2.72 \\
Flip & & & \\
Blink & 2.48 & 3.36 & 3.94 \\
Experiment 3 & 3.57 & 4.28 & 6.07 \\
$\quad$ Standard motion & 2.86 & 4.12 & 2.15 \\
Equiluminant motion & 4.29 & 5.01 & 6.13 \\
Standard blinking & & & \\
Equiluminant blinking & &
\end{tabular}

among-blinking, moving-among-moving, and movingamong-blinking) and set size $(9,17$, and 33$)$ as factors revealed main effects for condition $[F(3,33)=54.33$, $\left.M S_{\mathrm{e}}=381,141.92, p<.001\right]$ and set size $[F(2,22)=$ $\left.54.22, M S_{\mathrm{e}}=367,253.85, p<.001\right]$, as well as an interaction $\left[F(6,66)=13.48, M S_{\mathrm{e}}=200,445.95, p<.001\right]$. RTs were increased when all items were of one type of dynamics compared with when target and distractors were of different types of dynamics. Furthermore, RTs increased with set size in all conditions, but search slopes were steeper in the conditions in which all elements had the same type of dynamics, in comparison with those conditions in which target and distractor were of different types of dynamics. Table 2 contains the statistics for all pairwise comparisons between the conditions except for the set size main effects, which were significant for all comparisons (all $p$ s $<.001$ ). These comparisons show that the search slope in the blinking-among-moving condition differed from both the moving-among-moving and the blinking-among-blinking conditions. The same goes for the moving-among-blinking condition. Of further interest, the moving-among-blinking condition was reliably shallower than the search slope in the blinking-amongmoving condition, whereas the blinking-among-blinking condition did not differ from the moving-among-moving condition. Intercepts in the moving-among-blinking condition were somewhat higher than those in the blinkingamong-moving condition $[1,198$ vs. $905 \mathrm{msec}, t(11)=$ $3.74, p<.005]$, an effect that may be explained by assuming that abrupt onsets allowed for quicker processing in the response stage, in comparison with moving targets (Di Lollo, Enns, Yantis, \& Dechief [2000] indicate that this might be the case.)

Finally, we assessed whether search in the blinking condition was artificially improved by the fact that blinking distractors are invisible half of the time. For this purpose, we did the same analyses again, but used set sizes 17, 33 , and 65 in the conditions containing blinking distractors. These analyses are shown in Table 3 and reveal no major differences in the pattern of findings. Furthermore, a direct comparison of the blinking conditions with the standard set sizes $(9,17$, and 33) to those with double set sizes $(17,33$, and 65$)$ revealed only a significant increase in overall RTs $\left[F(1,11)=26.1, M S_{\mathrm{e}}=410,147.3, p<\right.$ $.001]$, but no difference in search slopes $(p s>.1)$. Note that with this double set size analysis, search slopes in the blinking-among-blinking condition were not linear; the search slope for set sizes 9 and 17 was higher than that for set sizes 17 and 33 [189 vs. $26 \mathrm{msec} / \mathrm{item}, t(11)=$ $3.20, p<.01]$. However, the flattening search slope was accompanied by higher error rates for set size 33 than for the other set sizes [ $22 \%$ vs. $12 \%, t(11)=5.20, p<.001]$, suggesting that, with the very dense displays, observers decided to cut short search at the expense of accuracy.

The main result of Experiment 1 is that search for a blinking target among moving distractors or for a moving target among blinking distractors is relatively efficient (26 and

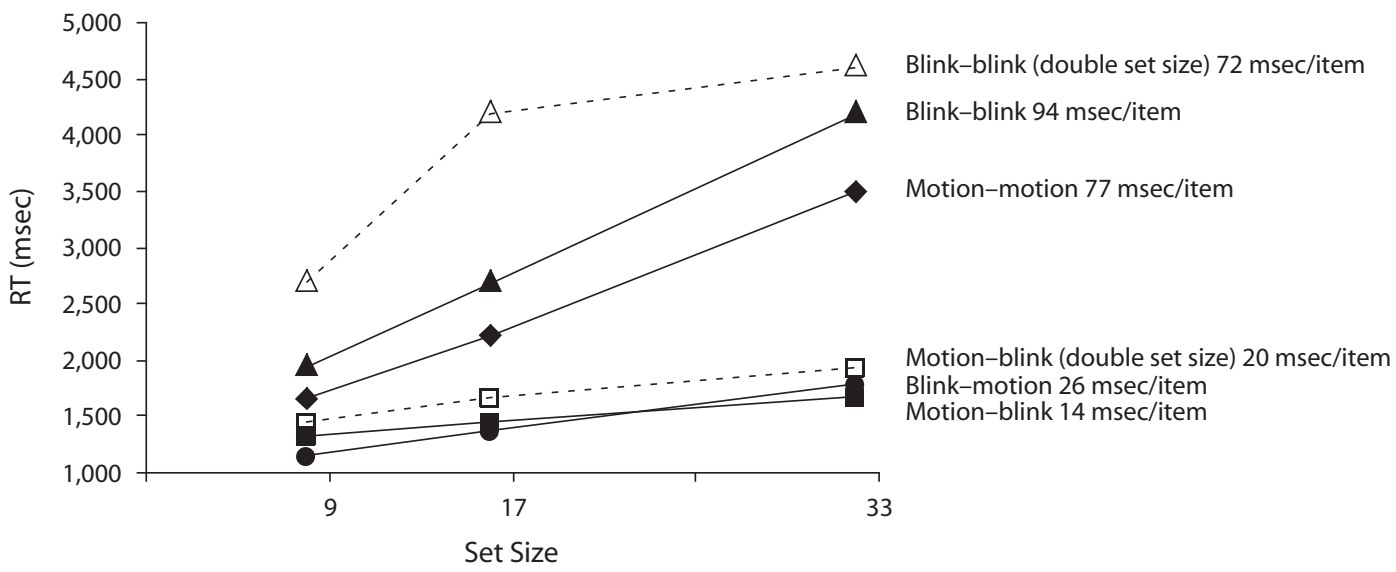

Figure 2. Mean response times (RTs) for each condition of Experiment 1 (moving-among-blinking, moving-amongmoving, blinking-among-moving, and blinking-among-blinking) as a function of set size. In addition to these, the results are displayed in dotted lines when each blinking distractor is counted as half a distractor. For each condition, the mean search slopes are provided. 
$14 \mathrm{msec} /$ item, respectively), whereas search for a moving target among moving distractors or a blinking target among blinking distractors is highly inefficient (77 and $94 \mathrm{msec} /$ item, respectively). This was the case even though the blinking and moving occurred at exactly the same frequencies and produced exactly the same luminance onsets and offsets. This finding refutes the broad division hypothesis, according to which attention can distinguish efficiently between static and dynamic channels, but not within these channels. ${ }^{1}$ The presence of a search asymmetry further corroborates this (cf. Treisman \& Gormican, 1988). Search for a moving target among blinking distractors was more efficient than that for blinking targets amoun moving distractors, suggesting that attention may find it easier to reject blinking distractors than moving distractors.

\section{EXPERIMENT 2 \\ Search for a Moving Target Among Moving and Blinking Distractors}

In Experiment 2, we wanted to assess whether we could further falsify the hypothesis that attention can only operate on a broad division between static and dynamic items. Participants searched for a target (a vertical or horizontal line) that moved in a random direction. All distractors (tilted lines) also moved in random directions. In the control condition, the distractors contained no other dynamic cues. In the two experimental conditions, the distractors possessed an additional dynamic cue. In one experimental condition, the distractors flipped back and forth between oblique orientations while moving, and in the other, they blinked (see Figure 3 for an example display). We employed different stimuli in Experiment 2 than in Experiment 1, since all items in Experiment 2 were moving translationally; therefore, adding another translational motion cue might not have been very helpful. The stimuli used in this experiment allowed for the addition of rotational motion cues (the flipping) on top of the translational motion.

If attention can only operate on a distinction between static and dynamic stimuli, then adding extra dynamic cues in an already fully dynamic environment should not improve search efficiency. Thus, according to the broad division hypothesis, search for the dynamic target should be equally inefficient among all types of dynamic distractors. If anything, the continuous flipping and blinking might only distract attention from the moving target.

Of greater importance is the question of what exactly it is that makes dynamic changes so useful to the attentional system. Pinto et al. (2006) found that search for a static item among distractors that abruptly changed luminance but did not disappear was much less efficient than when the distractors disappeared - even when the absolute and relative transients, in terms of luminance changes, were controlled for. This finding suggests that both luminance transients and object continuity can function as important cues in visual search (see also Abrams \& Christ, 2003; Kahneman, Treisman, \& Gibbs, 1992). If this is indeed the case, we expect search to be more efficient when the extra dynamic cue is blinking than when it is flipping, 


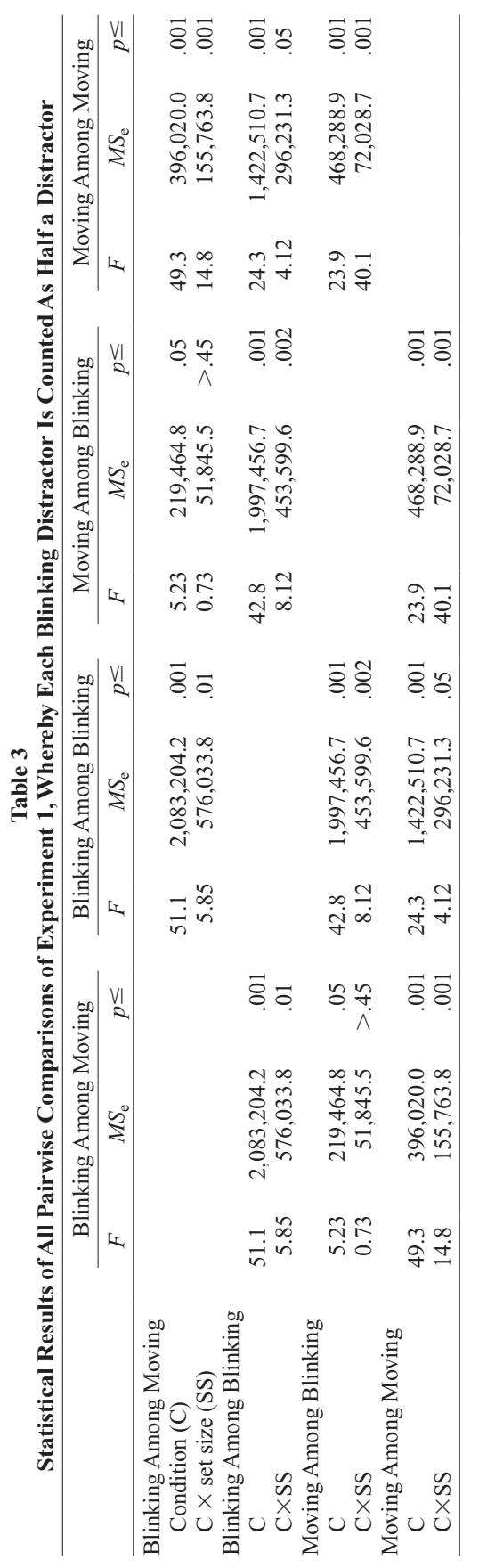

since in the blinking condition, the relative discontinuity of the distractors may provide an additional cue for segregating the background from the target. The search asymmetry found in Experiment 1 also suggests this.

\section{Method}

Participants. Nine participants ranging in age from 19 to 33 years, average 23.4 years, took part as paid volunteers. All participants completed all of the conditions. All had normal or correctedto-normal vision.

Apparatus, Stimuli, and Procedure. Everything was the same as in Experiment 1 except for the following changes. Participants searched for a nontilted line segment without a gap of size $0.70^{\circ}$, which could be either horizontal or vertical. The task of the participant was to indicate the orientation of the target. The distractors were the same as the target, but they were tilted $22.5^{\circ}$ to either side of the horizontal or vertical plane. The distractors could appear anywhere on an imaginary $7 \times 6$ matrix $\left(12.68^{\circ} \times 8.26^{\circ}\right)$. The target (horizontal or vertical) could appear anywhere except in the middle (row 4, column 3 or 4). Every element moved at a speed of $1.75^{\circ}$ visual angle/sec in a random direction. An item meeting the boundary of the display area would then continue its trajectory at the other end of the screen. The directions of the elements were uncorrelated. There were three conditions: motion-and-blink, motion-and-flip, and motion-only. In all conditions, the target moved in a random direction but underwent no other changes. In the motion-only condition, the distractors also moved in random directions and underwent no other changes. In the motion-and-flip condition, the distractors moved in random directions and flipped back and forth between their original orientation and $4^{\circ}$ arc deviation from this in either direction. The items flipped after a random period between 150 and $300 \mathrm{msec}$, as each had a $25 \%$ chance of flipping after $150 \mathrm{msec}, 25 \%$ after $200 \mathrm{msec}, 25 \%$ after $250 \mathrm{msec}$, and $25 \%$ after $300 \mathrm{msec}$. The chances of flipping were uncorrelated between the items and were uncorrelated to the previous time in between flips. In the motionand-blink condition, the distractors moved in a random direction and switched on and off, in the same way and at the same rate as in the motion-and-flip condition. In all conditions, set sizes varied randomly within a block between 9,17 , and 33 (i.e., 8,16 , or 32 distractors, plus one target). We no longer included set size 65 because Experiment 1 suggested that it added little to the results. The task was to determine the orientation of the target element. Participants pressed " $z$ " for vertical lines, and " $m$ " for horizontal lines. The experiment consisted of 15 blocks, each block containing 54 trials. The order of the blocks was determined according to a Latin square design. The first 3 blocks were disregarded as practice. The experiment took approximately $60 \mathrm{~min}$, with breaks between the blocks.

\section{Results and Discussion}

Overall, error percentages were low (see Table 1). A two-way ANOVA with condition (motion-only, motionand-flip, or motion-and-blink) and set size $(9,17$, or 33) as factors revealed a main effect for condition $[F(2,16)=$ 4.76, $\left.M S_{\mathrm{e}}=8.12, p<.05\right]$. Participants made the most errors in the motion-only condition. This pattern resembled the RT pattern, and a speed-accuracy trade-off could be excluded. We will therefore concentrate on RTs.

Trials on which RTs were 2.5 standard deviations away from the mean of the respective cell were excluded from analysis, resulting in a loss of approximately $3.5 \%$ of the trials. Figure 4 shows a graphical depiction of the results. A two-way ANOVA on mean RT for each participant with condition (motion-only, motion-and-flip, or motion-andblink) and set size $(9,17$, or 33$)$ as factors revealed a main effect for condition, as RTs were elevated in the motion- 


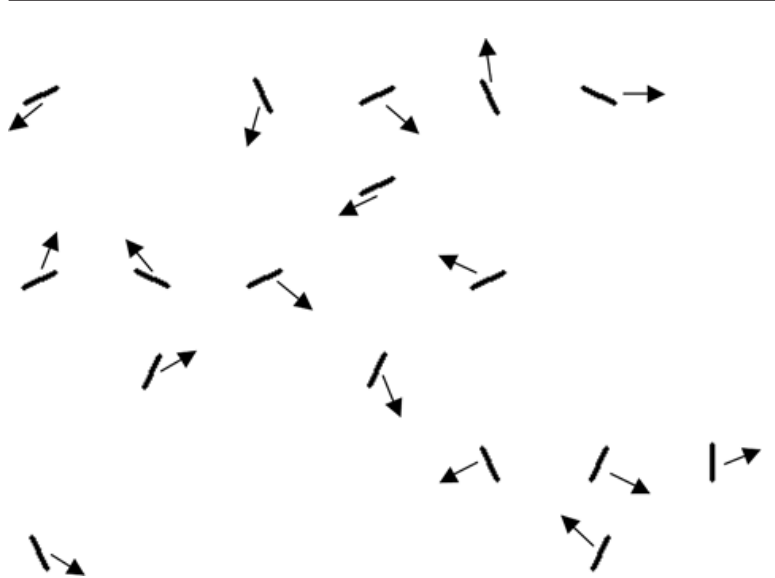

Figure 3. Typical example of the search display used in the control condition of Experiment 2. Participants searched for a vertical or horizontal line segment among slightly tilted line segments. In the actual experiments, the lines were white and the background was black. In this example, all items were moving in a random direction. Motion direction was uncorrelated between the items. In the motion-and-flip condition, in addition to moving, all distractors flipped back and forth between their original orientation and $a 5^{\circ}$ arc deviation from this in either direction, whereas the target only moved. In the motion-and-blink condition, the distractors switched on and off in addition to moving, whereas the target only moved.

only condition in comparison with the motion-and-flip and motion-and-blink conditions $\left[F(2,16)=21.87, M S_{\mathrm{e}}=\right.$ 490,817.67, $p<.001]$. Furthermore, RTs increased with set size $\left[F(2,16)=28.09, M S_{\mathrm{e}}=835,406.67, p<\right.$ $.001]$. There was also a significant interaction, reflecting the steeper search slope in the motion-only condition $\left[F(4,32)=16.04, M S_{\mathrm{e}}=165,237.32, p<.001\right]$ in comparison with the motion-and-flip and motion-and-blink conditions. Pairwise comparisons revealed that all conditions differed significantly from each other both in RTs and in search slopes. RTs were fastest and slopes shallowest in the motion-and-blink condition and were slowest and steepest in the motion-only condition [motion-only vs. motion-and-flip condition, $F(1,8)=19.23, M S_{\mathrm{e}}=$ $649,873.55, p<.005$; condition $\times$ set size, $F(2,16)=$ 15.18, $M S_{\mathrm{e}}=195,855.73, p<.001$; motion-only vs. motion-and-blink condition, $F(1,8)=24.76, M S_{\mathrm{e}}=$ $768,191.63, p=.001$; condition $\times$ set size, $F(2,16)=$ 18.38, $M S_{\mathrm{e}}=258,901.35, p<.001$; motion-and-flip vs. motion-and-blink condition, $F(1,8)=12.55, M S_{\mathrm{e}}=$ $54,387.84, p<.01$; condition $\times$ set size, $F(2,16)=5.36$, $\left.M S_{\mathrm{e}}=40,954.87, p<.05\right]$.

Experiment 2 shows that participants benefit from the additional dynamic information carried by the distractors when searching for an already dynamic target. This provides further evidence against the broad division hypothesis. Rather than just dividing between static and dynamic stimuli, attention can make use of specific types of dynamic information.

Note that although the target was dynamic (i.e., it moved in a randomly chosen direction), it was also relatively stable, in that it retained its integrity as an object. The distractors, on the other hand, apart from moving in random directions, abruptly changed at random moments, and may thus be regarded as less stable. The attentional system may make use of this relative object stability. Consistent with this, search was more efficient when the distractors switched on and off than when they remained present but flipped (i.e., showed apparent motion).

\section{EXPERIMENT 3 \\ Searching for a Static Target With and Without Luminance Differences}

A further way of trying to dissociate different types of dynamic properties with respect to their attention-guiding power is by using equiluminant stimuli. Previous research suggests that luminance plays an important role in motion detection (Cavanagh, Tyler, \& Favreau, 1984; Gegenfurtner \& Hawken, 1995). However, with regard to onset detection, luminance may be less important. Yantis and Hillstrom (1994) provided evidence that abrupt equiluminant onsets captured attention, whereas large luminance

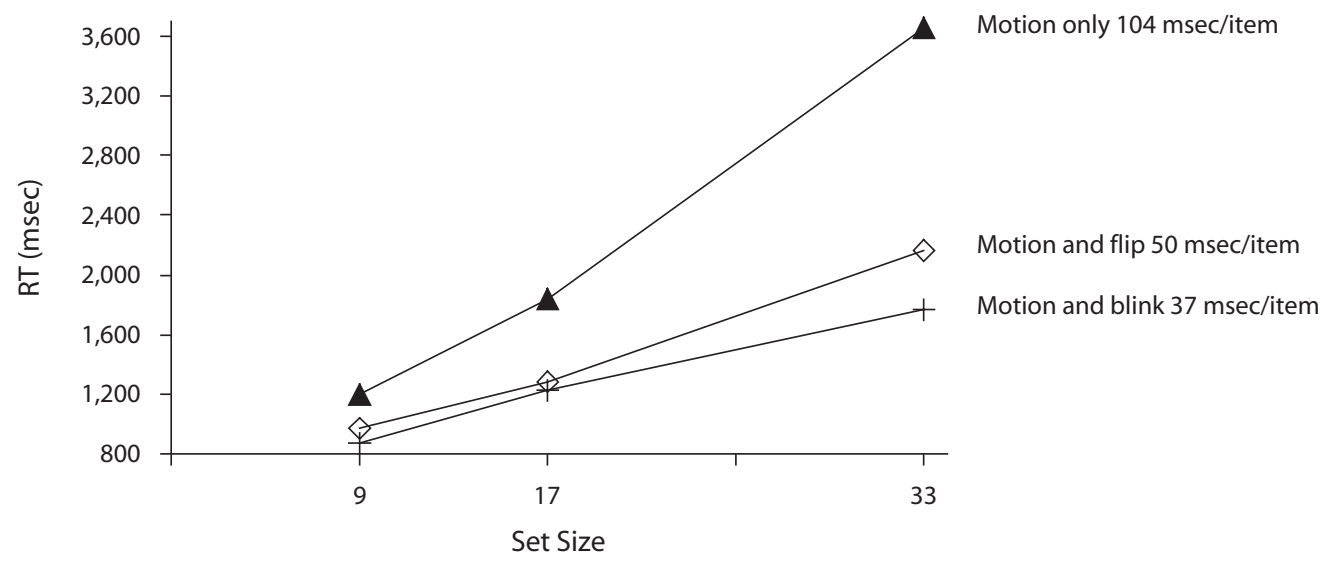

Figure 4. Mean response times (RTs) for each condition of Experiment 2 (motion-and-blink, motion-and-flip, and motion-only) as a function of set size. For each condition, the mean search slopes are provided. 
changes that did not signal the appearance of a new object did not (but see Theeuwes, 1995). They suggested that the appearance of a new object is crucial and that luminance change is not mandatory for onset detection. If motion detection is somehow dependent on luminance factors, but onset detection is not, we speculate that, under equiluminant conditions, the rejection of moving distractors may be impaired while the rejection of onset distractors will not.

\section{Method}

Participants. Twelve participants ranging in age from 18 to 25 years, average 20.8 years, took part as paid volunteers. All participants completed all of the conditions. All had normal or correctedto-normal vision.

Apparatus, Stimuli, Design, and Procedure. Everything was the same as in Experiment 1 except for the following changes. The target was always static, and the distractors were either moving or blinking (in the same way as in Experiment 1). Furthermore, participants were presented with two luminance conditions. In the standard conditions, the line segments were white and the background was black. In the equiluminant conditions, the line segments were green and the background was gray. Items and background were equiluminant. Fixed values for the colors were used, since a flicker fusion test (Ives, 1912) on several participants in pilot studies yielded very similar results. The CIE values were $x=0.298, y=0.607$, luminance $8.84 \mathrm{~cd} / \mathrm{m}^{2}$ for the green items; $x=0.281, y=0.283$, luminance $8.24 \mathrm{~cd} / \mathrm{m}^{2}$ for the gray background. In the standardblinking and standard-moving conditions, the targets were static, whereas the distractors were blinking or moving, respectively. The equiluminant-blinking and equiluminant-moving conditions were the same as the standard-blinking and standard-moving conditions, except now the items were presented green on gray instead of white on black. The experiment consisted of 20 blocks of 36 trials each. The order of the blocks was determined according to a Latin square design. The first 4 blocks were disregarded as practice. The experiment took approximately $60 \mathrm{~min}$, with breaks between the blocks.

\section{Results and Discussion}

Overall, error percentages were low (see Table 1). A three-way ANOVA with type of dynamics (motion, blinking), luminance condition (standard, equiluminant), and set size $(9,17$, or 33$)$ as factors revealed only a main effect for condition $\left[F(3,33)=2.94, M S_{\mathrm{e}}=0.001, p<.05\right]$. Overall, participants made more errors in the equiluminant conditions than in the standard conditions, a pattern that is in line with the RTs. For the remainder of the analyses, we will therefore concentrate on the RTs.

Trials on which RTs were 2.5 standard deviations away from the mean were excluded from analysis, resulting in a loss of approximately $4 \%$ of the trials. Figure 5 shows a graphical depiction of the results. A three-way ANOVA on mean RT for each participant with type of dynamics (motion, blinking), luminance condition (standard, equiluminant), and set size $(9,17$, or 33$)$ as factors revealed a main effect for type of dynamics $\left[F(1,11)=29.67, M S_{\mathrm{e}}=\right.$ $229,713.41, p<.001]$. RTs were higher in the motion than in the blinking condition. RTs also increased with set size $\left[F(2,22)=103.74, M S_{\mathrm{e}}=261,949.32, p<.001\right]$ and were slower for equiluminant stimuli $\left[F(1,11)=22.13, M S_{\mathrm{e}}=\right.$ $1,148,496.31, p=.001]$. There was a significant interaction between type of dynamics and set size $[F(2,22)=$ 23.26, $\left.M S_{\mathrm{e}}=95,640.92, p<.001\right]$, reflecting the overall steeper search slope in the motion condition than in the blinking condition. There was also a significant interaction between luminance condition and type of dynamics $\left[F(1,11)=5.17, M S_{\mathrm{e}}=210,391.34, p<.05\right]$; the luminance manipulation had a larger effect in the motion condition than in the blinking condition. Furthermore, there was a significant interaction between luminance condition and set size $\left[F(2,22)=6.11, M S_{\mathrm{e}}=152,783.34, p<.01\right]$, reflecting the overall steeper search slope in the equiluminance condition than in the standard condition. Most important, there was a three-way interaction between type of dynamics, set size, and luminance condition $[F(2,22)=$ $\left.9.48, M S_{\mathrm{e}}=74,169.62, p=.001\right]$, reflecting the fact that search efficiency in the motion condition was more affected by the equiluminance manipulation than search efficiency in the blinking condition was. Two separate ANOVAs with luminance and set size as factors revealed that the equiluminance manipulation had no effect on search slopes in the blinking condition $(F<1, p>.4)$, but did have a signifi-

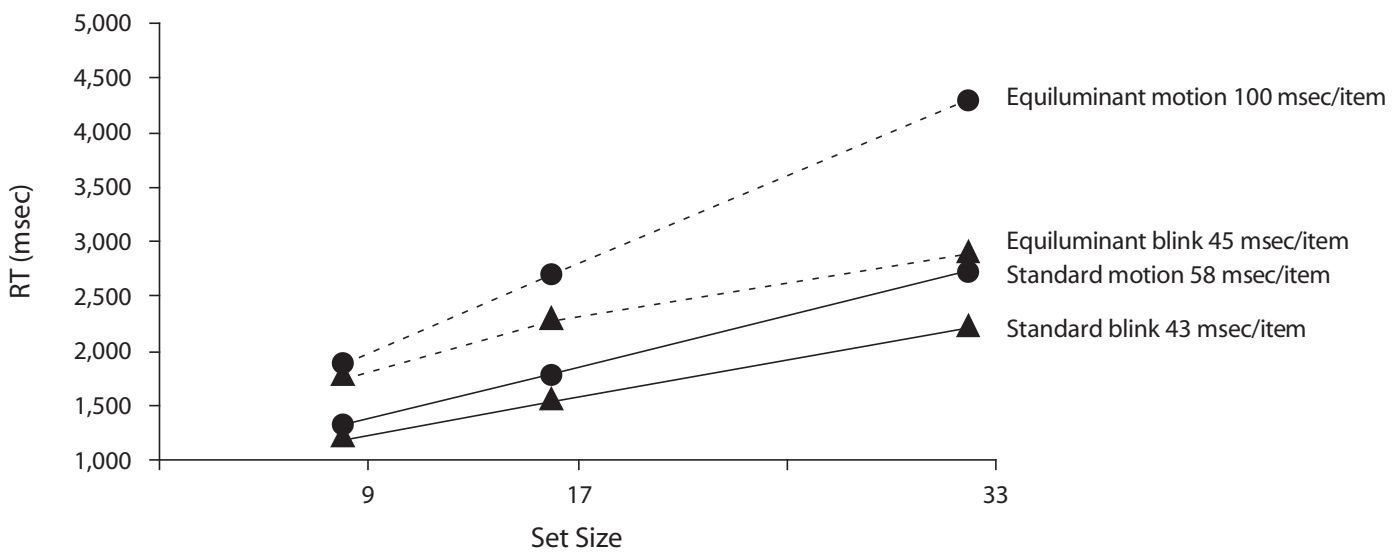

Figure 5. Mean response times (RTs) for each condition of Experiment 3 (standard-blinking, equiluminant-blinking, standard-moving, and equiluminant-moving) as a function of set size. For each condition, the mean search slopes are provided. 
cant effect in the motion condition $\left[F(2,22)=8.97, M S_{\mathrm{e}}=\right.$ $177,643.78, p=.001]$.

Experiment 3 shows that the rejection of moving distractors is impaired under equiluminant conditions. Importantly, the same did not hold for the rejection of blinking distractors. Blinking distractors were as efficiently rejected under equiluminant as under nonequiluminant circumstances. The results provide further evidence for a dissociation between the effects of different types of dynamic stimuli. The removal of (most) luminance cues differentially affects the rejection of moving and blinking distractors. This again implies that selection is not just based on a broad division between dynamic and static stimuli, but that selection depends on the exact type of dynamic stimulus (in this case, blinking vs. moving). Thus, the findings of Experiment 3 corroborate the results of the first two experiments and again suggest that onsets and moving stimuli are not treated alike by the attentional system. Furthermore, the results of Experiment 3 are congruent with the suggestion of Yantis and Hillstrom (1994), that onset detection is largely independent of luminance factors; but our findings are not conclusive support for their account, since Experiment 3 looked at how efficiently equiluminant onsets can be rejected rather than selected.

\section{GENERAL DISCUSSION}

Recently, we reported evidence that observers can relatively efficiently disregard dynamic distractors and find a static target, regardless of whether these distractors were moving or blinking (Pinto et al., 2006). Apparently, observers can make use of a broad division between static and dynamic channels and can selectively attend to one of them. Here we asked whether attention makes use only of this broad division or makes specific distinctions between different types of dynamic changes.

In Experiment 1, we found that a difference in type of change between the target and the distractors could guide search, even though the abruptness, frequency, and local luminance increase of the changes was the same for both types. This goes against the broad division hypothesis. Search was efficient for blinking targets among moving distractors, as well as for moving targets among blinking distractors, but with a slight advantage for the latter condition. This search asymmetry between the two conditions provides further evidence for a distinction between the two types of dynamics.

In Experiment 2, all display items continuously moved around in random directions. Hence, the entire display was dynamic. Nevertheless, it was shown that additional dynamic cues (i.e., blinking or flipping distractors) improved search, again suggesting that attention can make use of more information than is provided by a broad static/ dynamic division. Here too, blinking distractors were more easily ignored than flipping distractors.

Finally, Experiment 3 revealed that, under equiluminant conditions, blinking distractors could be as efficiently ignored as under high contrast conditions, whereas the rejection of distractors featuring apparent motion suffered from the equiluminance. This provides further evidence for dissociations in attentional processing of different types of dynamic stimuli.

\section{Object Continuity}

The findings point toward object continuity as an important factor. In Experiment 1, a moving but continuously present target was more efficiently found between abruptly onsetting and offsetting distractors, than were onsetting and offsetting targets among moving and continuously present distractors. The same was true in the standard conditions of Experiment 3 for static targets among blinking distractors, in comparison with those among moving distractors. This may be because moving distractors are regarded as being more continuous than the disappearing distractors. Such continuous distractors may then be regarded as stronger competitors in search for a continuous target. In Experiment 2, the target itself was dynamic (i.e., it was moving), but it was also relatively continuous in that, unlike the distractors, it did not abruptly change. Again search was aided by changing distractors, and again search was aided a little more when the distractors were blinking rather than flipping - the latter presumably providing more continuity. Finally, under the equiluminant conditions of Experiment 3, the presumably more continuous moving distractors were relatively even more difficult to ignore, whereas the appearing and disappearing blinking distractors allowed for search to be as efficient as in the standard baseline with luminance differences.

Further support for object continuity playing an important role in visual search of dynamic displays comes from Experiment 5 of Pinto et al. (2006). In that experiment, participants searched for a static target among distractors that changed in luminance. The distractors could disappear as a result of the luminance change or remain on the screen. In both cases, the luminance change was equally large. However, search was efficient only when offset was complete. Pinto et al.'s results neatly fitted those of Davis and Leow (2005), who found inefficient search for a static target among distractors that changed luminance and color, but did not disappear. Both findings suggest that, when both target and distractors are relatively continuous (i.e., changing, but not disappearing), search is less efficient than when one of the two loses its continuity (i.e., disappearing).

Further evidence that object continuity plays a role in visual search comes from several other studies. Yantis and Hillstrom (1994) employed a visual search task in which, in one condition, the target underwent an abrupt luminance change, and in another condition, the target appeared as an equiluminant onset. They found that luminance changes did not capture attention, whereas equiluminant onsets did. Again, it seems that a sudden discrepancy in the integrity of an object is more important to the attentional system than a physically equally large change to a continuously displayed object is. Enns, Austen, Di Lollo, Rauschenberger, and Yantis (2001) reported a similar finding. They found that new objects were more effective in guiding search than luminance changes to old objects, even if the luminance change defining the onset was smaller than the luminance change to the old object. Furthermore, Cole, Kentridge, and Heywood (2005) found that the appearance of a new object captures attention in visual search, whereas a change of color to an existing object does not. All these findings support the notion that violations of object continuity are important to the attentional system. Finally, object continuity has also been 
shown to affect nonsearch tasks, such as multiple object tracking (Scholl \& Pylyshyn, 1999) and object matching tasks (Mitroff, Scholl, \& Wynn, 2004). This is not to say that luminance changes per se are not important. Franconeri, Hollingworth, and Simons (2005) used dynamic occlusion to show that, under these circumstances, new objects only capture attention when they are accompanied by large luminance changes. This shows that object continuity and luminance information interact: New object onsets make luminance changes more effective, and vice versa.

\section{Attention Based on Object Files}

An explanation for why object continuity plays such an important role for the attentional system might come from the object file hypothesis. Kahneman, Treisman, and Gibbs (1992) suggested that the visual system is organized on the basis of objects. According to this theory, a separate file is created for each object. Whenever something changes within an existing object, the file is updated. The appearance of a new object leads to the creation of a new object file. Thus, it is the appearance of a new object that is most relevant to the attentional system. Within this hypothesis, object continuity becomes crucial. Continuously existing objects only lead to updating of existing object files, whereas severe discontinuities in an object's spatiotemporal landscape cause the creation or deletion of object files (see also Mitroff et al., 2004; Yantis \& Gibson, 1994).

According to the object file hypothesis, new object files require attention to be formed (Kahneman et al., 1992). Perhaps, then, large discontinuities - like abrupt onsetscapture attention to make the creation of such a file possible (cf. Yantis, 1998). If the object concerned is the target, then performance will benefit from the attentional capture caused by the discontinuity.

However, there are several problems with the object file explanation of the present findings. First, according to Kahneman et al. (1992), only three to four object files can be maintained at any one time. In the present experiments, there were 8 to 32 distractors, implying that most of the distractors were necessarily unaffected by the existence (or disappearance) of object files. Second, note that in our experiments, the discontinuous objects were the distractors. If such discontinuities indeed automatically demand the creation of a new object file for each of these distractors, then we should expect rather inefficient search as the new distractors continuously draw attention away.

With respect to the second objection, the work of von Mühlenen et al. (2005) might be important. Their findings suggest that temporal uniqueness of change determines whether a changing item captures attention. They found that, when a change to an item occurred when no other items were changing, the item was more effective in capturing attention. In our displays, with up to 32 randomly blinking distractors, a single distractor rarely produced a temporally unique onset. In such cases, then, single distractors are less effective attentional captors.

Another possibility is that new object files indeed need attention to be created, but that this is not an automatic process. Perhaps, at the start of the display, object files are created for all the items in the display (distractors and target alike). When a distractor then briefly disappears, its object file is destroyed. It is not recreated when the distractor reappears. After a few rounds of disappearances, the target remains as the only one with an intact object file, allowing for relatively efficient search.

A similar suggestion could also account for the first objection. Search for the only static object is efficient not despite the limited number of object files, but because of the limited number of object files. Perhaps due to the limited amount of possible object files, unstable objects do not get object files assigned to them. This then would leave the static object as the only item with an object file attached to it, which would allow for efficient search.

\section{Temporal Synchrony}

Another possibility is that it is not so much the individual qualities of the distractors that allowed for efficient search, but their global properties. According to the temporal synchrony account (Engel \& Singer, 2001; Jiang, Chun, \& Marks, 2002), elements that carry the same temporal properties are grouped together. Perhaps the distractors in these displays are not treated as individual objects, but are grouped into a single dynamic surface, with a single object file attached. This grouping may occur despite the randomness with which each item changes (i.e., they do not change in synchrony). Merely the fact that they are all dynamic may cause them to be grouped together. Actually, phenomenologically, the displays resemble a water surface glistening in the sun. The target then represents the "discontinuity" to this surface. As the single static item, it breaks the dynamics, like a pole standing out of the glistening water surface.

\section{Fine Tuning of Attentional Control}

Davis and Leow (2005) suggested that a static target can be efficiently found among moving, but not among blinking distractors. Pinto et al. (2006) showed that this is not the case, and, that among both types of distractors, static targets can be found efficiently. The present research disproves Davis and Leow's suggestion even more strongly. In all the present experiments, the static target was more efficiently found among blinking than among moving distractors.

The findings of the present study also seem at odds with the conclusions of Folk et al. (1994). In their study, it appeared that participants could only set their attentional control to either static or dynamic, whereas our findings indicate that within the dynamic domain, subtler settings can be adopted. An explanation of this discrepancy might be along the lines of the attentional weighting account of Müller, Heller, and Ziegler (1995). According to this account, attentional weights are distributed over different dimensions, such as color, motion, and form. Similar weighting might occur within a certain dimension to particular values - for instance, within the color dimension, they might be differentially distributed between blue and red. Such a weighting account would suggest that the manner in which fine attentional control is tuned depends on the task at hand. In the first three experiments of Folk et al., the target could be either static or dynamic. Within 
this multidimensional task setting, attention may be set only to either the static or the dynamic dimension. A static precue may then not interfere with dynamic targets, nor vice versa, whereas different types of dynamic stimuli may interfere with each other. Going against this though are the last two experiments of Folk et al., in which only dynamic targets were employed, but attention was still not set to particular values within the dynamic dimension (i.e., motion or onsets). However, note that in Folk et al.'s experiments, there may have been no real need to adopt an attentional set for a more specific dynamic property. In all their target displays, the target was a dynamic item among static distractors, regardless of which specific type of dynamics was used. Hence, it would be sufficient for observers to merely adopt a more general attentional set for dynamic targets. In our experiments, more specific control settings were sometimes needed and possiblefor example, as with search for a blinking target among moving distractors in Experiment 1, the task set allows for specific settings, and efficient search makes these specific settings necessary. Therefore, in the present study, we did find a more fine-tuned attention setting within the dynamic domain.

Finally, a related question here is whether moving and blinking should be regarded as separate dimensions, or whether one is a subdimension of the other. Several researchers have described the detection of blinking items as a form of higher order motion detection (e.g., Nishida \& Sato, 1994; O'Keefe \& Movshon, 1998). This might suggest that blinking is a subcategory of motion; that is, that blink detection is a specialized form of motion detection. Experiment 3 of the present study casts doubt on this notion. In Experiment 3, rejection of moving stimuli was impaired under the equiluminant condition, whereas rejection of blinking stimuli was not affected. This suggests that flicker detection is not a subcategory of motion detection.

\section{CONCLUSION}

The present research shows that, in search for a static object in a dynamic environment, attentional control is not restricted to a broad division between dynamic and static stimuli. Within the dynamic domain, attention can be specifically set to certain types of change. Furthermore, object continuity and task demands probably play an important role in explaining these results. Finally, the present work suggests that onset detection is not a specialized form of motion detection.

\section{AUTHOR NOTE}

Correspondence concerning this article should be addressed to Y. Pinto, Department of Cognitive Psychology, Vrije Universiteit, Van der Boechhorststraat 1, 1081 BT Amsterdam, The Netherlands (e-mail: y.pinto@psy.vu.nl).

\section{REFERENCES}

Abrams, R. A., \& Christ, S. E. (2003). Motion onset captures attention. Psychological Science, 14, 427-432.

Albright, T. D. (1984). Direction and orientation selectivity of neurons in visual area MT of the macaque. Journal of Neurophysiology, 52, 1106-1130.
Andersen, R. A. (1997). Neural mechanisms of visual motion perception in primates. Neuron, 18, 865-872.

Breitmeyer, B. G., \& Ganz, L. (1976). Implications of sustained and transient channels for theories of visual pattern masking, saccadic suppression, and information processing. Psychological Review, 83, 1-36.

Cavanagh, P., Tyler, C. W., \& Favreau, O. E. (1984). Perceived velocity of moving chromatic gratings. Journal of the Optical Society of America A, 1, 893-899.

Chapman, C., Hoag, R., \& Giaschi, D. (2004). The effect of disrupting the human magnocellular pathway on global motion perception. $\mathrm{Vi}$ sion Research, 44, 2551-2557.

Cole, G. G., Kentridge, R. W., \& Heywood, C. A. (2005). Object onset and parvocellular guidance of attentional allocation. Psychological Science, 16, 270-274.

Davis, G., \& LeOw, M. C. (2005). Blindness for unchanging targets in the absence of motion filtering: A response to Theeuwes (2004). Psychological Science, 16, 80-82.

Di Lollo, V., Enns, J. T., Yantis, S., \& Dechief, L. G. (2000). Response latencies to the onset and offset of visual stimuli. Perception \& Psychophysics, 62, 218-225.

Engel, A. K., \& Singer, W. (2001). Temporal binding and the neural correlates of sensory awareness. Trends in Cognitive Sciences, $\mathbf{5}$, 16-25.

Enns, J. T., Austen, E. L., Di Lollo, V., Rauschenberger, R., \& YANTIS, S. (2001). New objects dominate luminance transients in setting attentional priority. Journal of Experimental Psychology: Human Perception \& Performance, 27, 1287-1302.

Folk, C. L., Remington, R. W., \& Wright, J. R. (1994). The structure of attentional control: Contingent attentional capture by apparent motion, abrupt onset, and color. Journal of Experimental Psychology: Human Perception \& Performance, 20, 317-329.

Franconeri, S. L., Hollingworth, A., \& Simons, D. J. (2005). Do new objects capture attention? Psychological Science, 16, 275-281.

Franconeri, S. L., \& Simons, D. J. (2003). Moving and looming stimuli capture attention. Perception \& Psychophysics, 65, 999-1010.

Gegenfurtner, K. R., \& Hawken, M. J. (1995). Temporal and chromatic properties of motion mechanisms. Vision Research, 35, 1547-1563.

Gottlieb, J. P., Kusunoki, M., \& Goldberg, M. E. (1998). The representation of visual salience in monkey parietal cortex. Nature, 391, 481-484.

Green, M. (1981). Psychophysical relationships among mechanisms sensitive to pattern, motion and flicker. Vision Research, 21, 971-983

Hillstrom, A. P., \& Yantis, S. (1994). Visual motion and attentional capture. Perception \& Psychophysics, 55, 399-411.

IvES, H. E. (1912). Studies of the photometry of different colours: I. Spectral luminosity curves obtained by equality of brightness photometer and the flicker photometer under similar conditions. Philosophical Magazine, 24(Series 6), 149-188.

JiAng, Y., Chun, M. M., \& Marks, L. E. (2002). Visual marking: Selective attention to asynchronous temporal groups. Journal of Experimental Psychology: Human Perception \& Performance, 28, 717-730.

Kahneman, D., Treisman, A., \& Gibbs, B. J. (1992). The reviewing of object files: Object-specific integration of information. Cognitive Psychology, 24, 175-219.

Livingstone, M. [S.], \& Hubel, D. [H.] (1988). Segregation of form, color, movement, and depth: Anatomy, physiology, and perception. Science, 240, 740-749.

MCLEOD, P., Driver, J., \& CRISP, J. (1988). Visual search for a conjunction of movement and form is parallel. Nature, 332, 154-155.

Mitroff, S. R., Scholl, B. J., \& WynN, K. (2004). Divide and conquer: How object files adapt when a persisting object splits into two. Psychological Science, 15, 420-425.

Müller, H. J., Heller, D., \& Ziegler, J. (1995). Visual search for singleton feature targets within and across feature dimensions. Perception \& Psychophysics, 57, 1-17.

Müller, H. J., \& vON MüHLEnEN, A. (1999). Visual search for conjunctions of motion and form: The efficiency of attention to static versus moving items depends on practice. Visual Cognition, 6, 385-408.

Nishida, S., \& Sato, T. (1994). Motion aftereffect with flickering test patterns reveals higher stages of motion processing. Vision Research, 35, 477-490. 
O'Keefe, L. P., \& Movshon, J. A. (1998). Processing of first- and secondorder motion signals by neurons in area MT of the macaque monkey. Visual Neuroscience, 15, 305-317.

Pinto, Y., Olivers, C. N. L., \& Theeuwes, J. (2006). When is search for a static target among dynamic distractors efficient? Journal of Experimental Psychology: Human Perception \& Performance, 32, 59-72.

Scholl, B. J., \& Pylyshyn, Z. W. (1999). Tracking multiple items through occlusion: Clues to visual objecthood. Cognitive Psychology, 38, 259-290.

TheEUwes, J. (1995). Abrupt luminance change pops out; abrupt color change does not. Perception \& Psychophysics, 57, 637-644.

Theeuwes, J. (2004). No blindness for things that do not change. Psychological Science, 15, 65-70.

Treisman, A., \& Gormican, S. (1988). Feature analysis in early vision: Evidence from search asymmetries. Psychological Review, 95, 15-48.

von Mühlenen, A., Rempel, M. I., \& Enns, J. T. (2005). Unique temporal change is the key to attentional capture. Psychological Science, 16, 979-986.

Watson, D. G., \& Humphreys, G. W. (1995). Attention capture by contour onsets and offsets: No special role for onsets. Perception \& Psychophysics, 57, 583-597.

YANTIS, S. (1998). Control of visual attention. In H. Pashler (Ed.), Attention (pp. 223-256). San Diego: Psychology Press.

Yantis, S., \& Gibson, B. S. (1994). Object continuity in apparent motion and attention. Canadian Journal of Experimental Psychology, 48, 182-204.
Yantis, S., \& Hillstrom, A. P. (1994). Stimulus-driven attentional capture: Evidence from equiluminant visual objects. Journal of Experimental Psychology: Human Perception \& Performance, 20, 95-107.

YANTIS, S. \& JonidES, J. (1984). Abrupt visual onsets and selective attention: Evidence from visual search. Journal of Experimental Psychology: Human Perception \& Performance, 10, 601-621.

ZEKI, S. M. (1974). Functional organization of a visual area in the posterior bank of the superior temporal sulcus of the rhesus monkey. Journal of Physiology, 236, 549-573.

Zeki, S. [M.], Watson, J. D. G., Lueck, C. J., Friston, K. J., KenNARD, C., \& FRACKOWIAK, R. S. J. (1991). A direct demonstration of functional specialization in human visual cortex. Journal of Neuroscience, 11, 641-649.

Zihl, J., VON CRAMON, D. Y., \& MAI, N. (1983). Selective disturbance of movement vision after bilateral brain damage. Brain, 106, 313-340.

\section{NOTE}

1. Both Experiment 1 and Experiment 3 have been replicated using rotational instead of lateral motion. In the replication of Experiment 3 for each participant different equiluminance values were used. These values were determined for each participant through a flicker fusion test (Ives, 1912). These experiments yielded essentially the same results as the experiments reported in the present article.

(Manuscript received August 17, 2006; revision accepted for publication July 18, 2007.) 